\title{
Does the Cage Position in Transforaminal Lumbar Interbody Fusion Determine Unilateral versus Bilateral Screw Placement?: A Review of the Literature
}

\author{
Haley M. McKissack ${ }^{1}$, Howard Benjamin Levene ${ }^{2}$ \\ ${ }^{1}$ Herbert Wertheim College of Medicine, Florida International University, Miami, FL, USA \\ ${ }^{2}$ Department of Neurological Surgery, Miller School of Medicine, University of Miami, Miami, FL, USA
}

\begin{abstract}
This literature review examines the relative placement of the interbody cage with respect to the unilateral screw construct to address the need for bilateral screw placement versus unilateral screw placement. Transforaminal lumbar interbody fusion (TLIF) has become a widely used technique for correcting lumbar intervertebral pathologies. This review addresses the necessity for further study on the effects of the relative position of intervertebral cage placement on the outcome of lumbar spine surgery after TLIF with unilateral pedicle screw fixation. Previous studies have addressed various factors, including posterior screw fixation, cage size, cage shape, and number of levels fused, that impact the biomechanics of the lumbar spine following TLIF. A simple survey of the literature was conducted. A search of the English literature was conducted using the keywords 'TLIF,' 'transforaminal lumbar interbody fusion,' 'graft placement,' 'graft position,' 'cage position,' 'cage placement,' 'unilateral pedicle screw,' 'unilateral TLIF cage placement,' 'lumbar biomechanics,' 'lumbar stability, ' 'lumbar fusion,' and 'lumbar intervertebral cage' with various combinations of the operators 'AND' and ' $\mathrm{OR}$ ' and no date restrictions. Seventeen articles in the English literature that were most relevant to this research question were identified. To the best of our knowledge, there are no published data addressing the effects of cage placement relative to the unilateral screw on lumbar stability in TLIF with unilateral pedicle screw fixation. Investigation of the effects of cage placement is, thus, warranted to achieve optimal clinical outcomes in patients undergoing TLIF with unilateral pedicle screw fixation.
\end{abstract}

Keywords: Lumbar vertebrae; Pedicle screws; Spinal fusion; Bone screws; Review

\section{Introduction}

Transforaminal lumbar interbody fusion (TLIF) is a surgical technique used for the treatment of symptomatic intervertebral disk pathologies. In intervertebral body fusions, the intervertebral disk is partially or almost fully removed, and one or more cages containing graft mate- rial are placed in the intervertebral space. The cage serves to maintain the intervertebral height and lumbar lordosis [1] while allowing vertebral bone ingrowth and fusion [2]. Adequate intervertebral rigidity is necessary for graft fusion without dislodgment [3] and is therefore essential to patient recovery [4]. To further stabilize the fusion and promote restoration of lordosis, posterior instrumentation

Received Apr 13, 2018; Revised Jul 6, 2018; Accepted Jul 25, 2018

Corresponding author: Howard Benjamin Levene

Department of Neurological Surgery, The Miami Project to Cure Paralysis, Miller School of Medicine, University of Miami, Lois Pope Life Center-2nd Floor, 1095 NW 14th Terrace (D4-6), Miami, FL 33136 USA

Tel: +1-305-575-7059, Fax: +1-305-243-3337, E-mail: hlevene@med.miami.edu 
is utilized by insertion of unilateral or bilateral pedicle screws [3]. TLIF has become an increasingly popular fusion technique due to its anatomical advantages, which reduce damage to the thecal sac and spinal nerve roots and preserve contralateral structures [5]. Multiple systematic reviews and meta-analyzes have reported TLIF fusion rates ranging from $89.71 \%$ to $97.53 \%[5,6]$.

In TLIF procedures, bilateral pedicle screws are traditionally utilized as additional posterior fixation because they provide the greatest stability. However, although its efficacy compared with bilateral fixation is debated, unilateral pedicle screw placement may be more desirable because it is less invasive and entails less operative time, less blood loss $[7,8]$, and less destruction of posterior structures $[1,7,9]$. In addition, bilateral fixation may increase stress at adjacent intervertebral levels in patients undergoing multi-level fusion due to increased stiffness, which can ultimately lead to degeneration $[7,10,11]$.

Some studies comparing TLIF outcomes with unilateral and bilateral pedicle screw fixation have reported no significant differences in fusion rates [5,7,9], spinal stability [8], and long-term clinical outcomes [7]. However, other studies suggest that unilateral screw fixation provides insufficient stability and jeopardizes healing $[11,12]$. To the best of our knowledge, the effect of cage placement relative to the screw for TLIF with unilateral pedicle screw stabilization has not been studied. It is thus worthwhile to investigate whether cage placement position impacts the need to use a contralateral screw, which is a more invasive method of posterior fixation.

Placement is important when considering the different sizes and shapes of cages. In the TLIF approach, a unilateral facetectomy is performed on the symptomatic side, decompressing the exiting spinal nerve root and allowing access to the intervertebral space [11]. Because of the restricted access space in TLIF, cages with a smaller footprint are warranted yet may be associated with increased complication rates [13]. Smaller footprints in the central part of the vertebral endplate may be more likely to subside [4] and experience fusion failure at lower loads [14]. Consideration of cage placement in TLIF within the disk space relative to the unilateral fusion may therefore be essential when aiming for optimal stability.

To implement the most appropriate construct for optimal lumbar stability, it is important to evaluate the stabilization effects of cage position on TLIF as it pertains to screw fixation and cage shape. Such a strategy is expected to result in improved surgical outcomes. To the best of our knowledge, no studies have clearly elucidated the significance of the placement of the interbody cage on the stability of the lumbar spine in TLIF procedures with unilateral pedicle screw fixation. The purpose of this review was thus to address the necessity of determining the structural effects of cage placement on the lumbar spine in TLIF. Ultimately, this will determine whether cage position can be manipulated to affect the TLIF construct such that stability with unilateral pedicle screw fixation is equivalent to that of TLIF with bilateral fixation.

\section{Materials and Methods}

A simple survey of the literature was conducted. A PubMed search was conducted with the keywords 'TLIF, 'transforaminal lumbar interbody fusion,' 'graft placement,' 'graft position,' 'cage position,' 'cage placement,' 'unilateral pedicle screw,' 'unilateral TLIF cage placement,' 'lumbar biomechanics,' 'lumbar stability,' 'lumbar fusion, and 'lumbar intervertebral cage' in various combinations using the operators 'AND' and 'OR.' The language was restricted to English, and articles were cross-referenced. No date restrictions were used.

\section{Results}

As summarized in Table 1 [1-13,15-17], the search resulted in one systematic review with meta-analysis, two metaanalyzes, three randomized control trials, two retrospective cohort studies, four in vivo biomechanical studies, one in vitro biomechanical study and narrative review, two narrative reviews, and one technical description. There were no prospective double blinded studies and no studies directly addressing the question of placement of the interbody device relative to the unilateral pedicle screw.

\section{Discussion}

Previous studies of the relationship between the position of the interbody cage and the effect of stability on the lumbar spine have presented conflicting results [16]. Ames et al. [3] compared the stabilities of posterior lumbar interbody fixation and TLIF using three separate approaches: the difference in range of lumbar motion after one or two level TLIF without pedicle screw fixation, the additional stability provided by bilateral pedicle screw fix- 


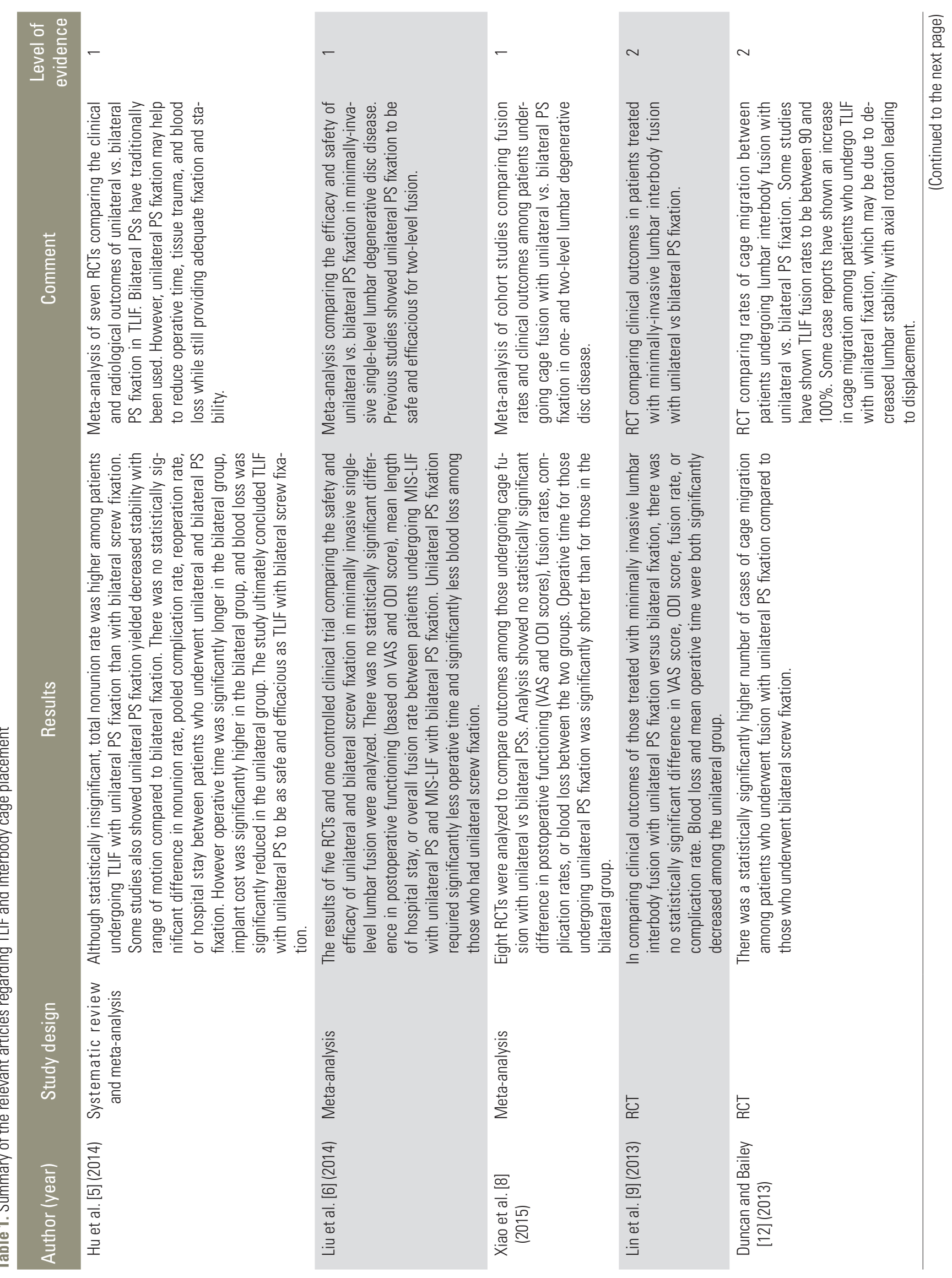




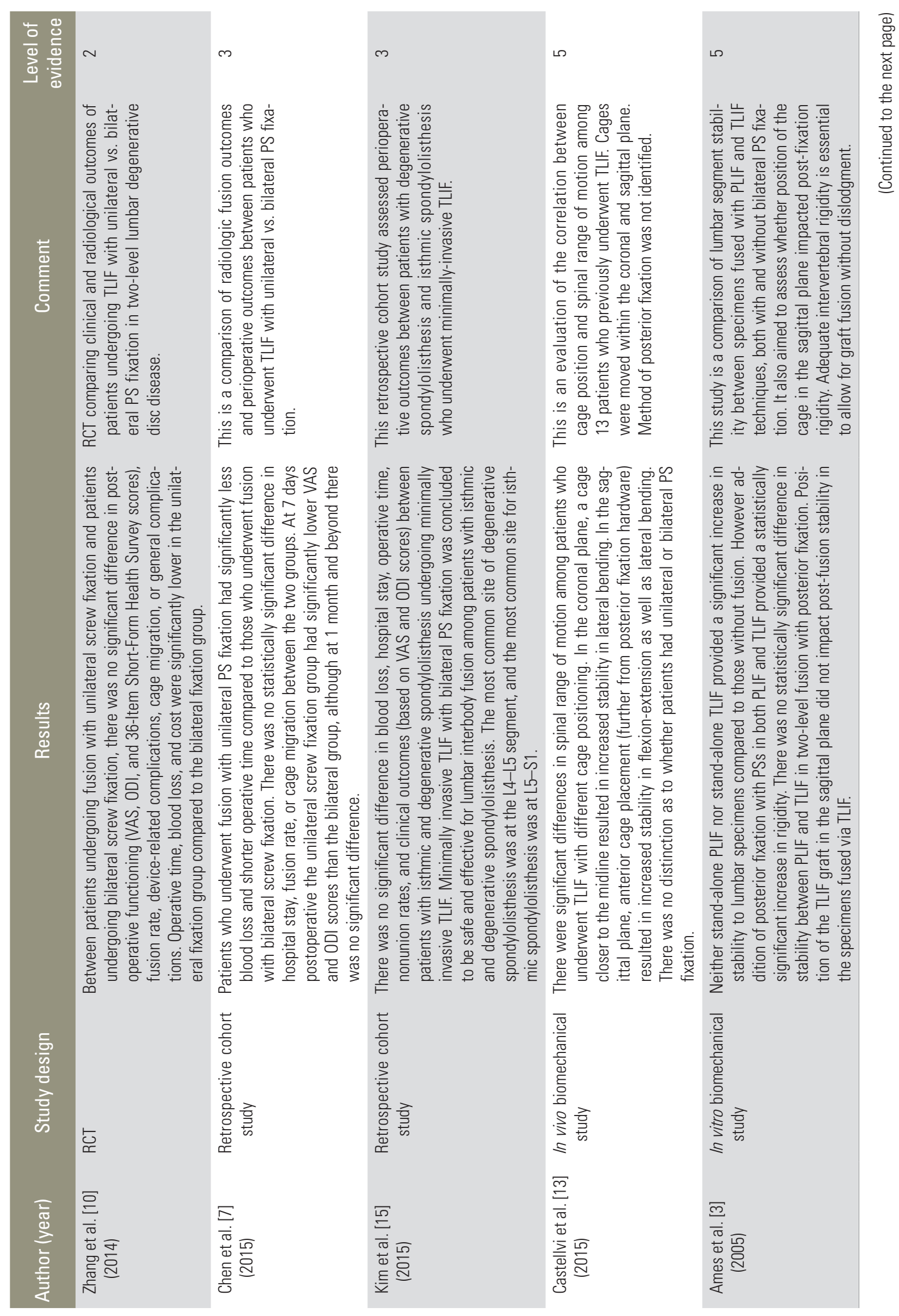




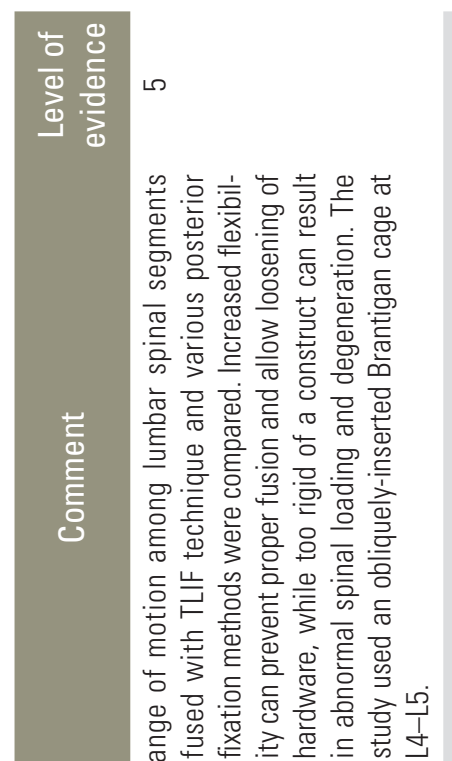

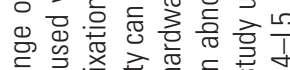
究

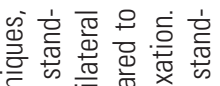

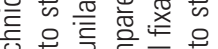

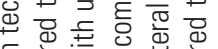

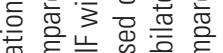

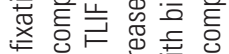

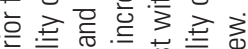

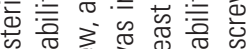

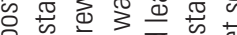

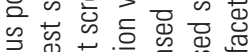

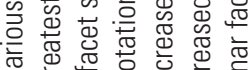

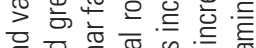
完

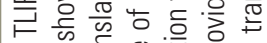

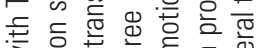

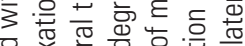

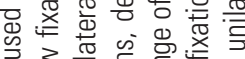

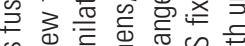
禹娄

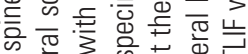

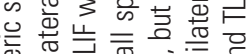

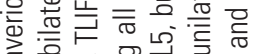

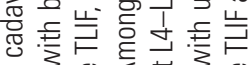

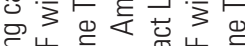

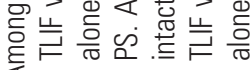

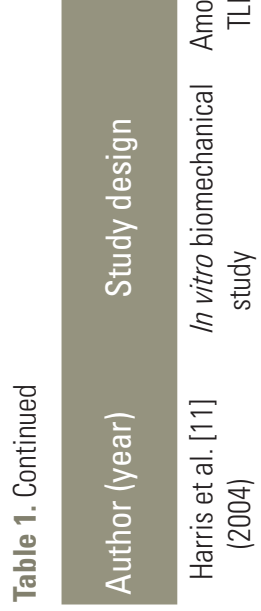

\llcorner

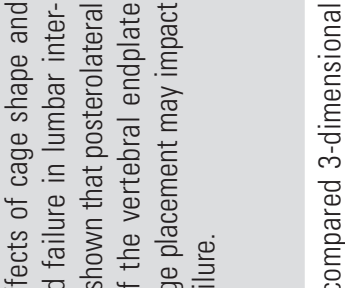

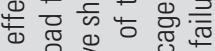

Ð

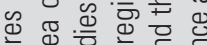

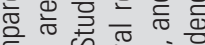

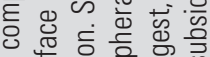

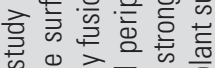

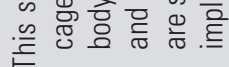

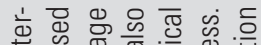

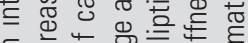
ธ

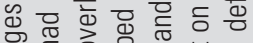
गु तु

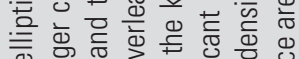
믄 出 त

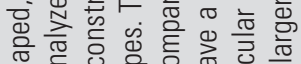

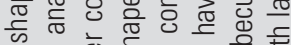

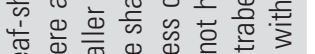

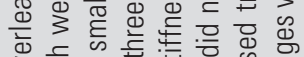

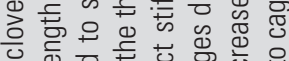

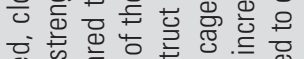

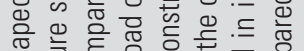
穃 》ั 需 ๘

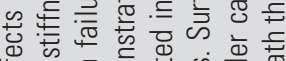

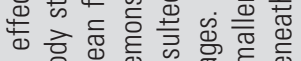
$\stackrel{\Phi}{\risingdotseq}$

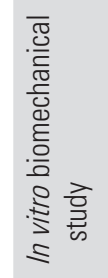

导

त्र

용

เ
즌

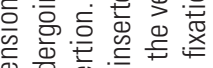

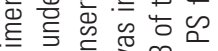

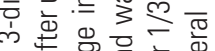
유 윰

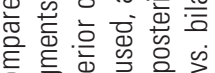

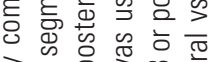
立.

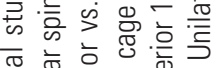

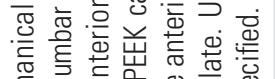

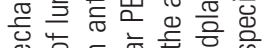

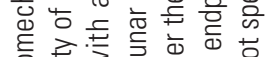

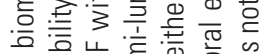

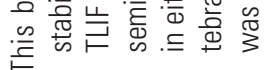

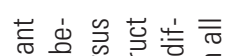

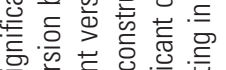

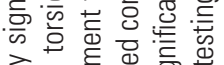
专䨌 .리인

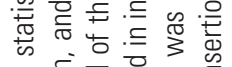

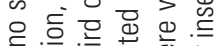

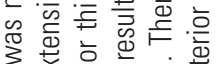
(1) 응 도

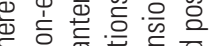

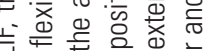
히.든 능.은

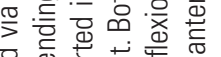

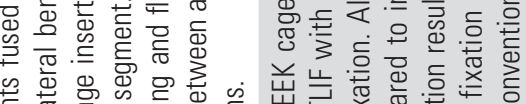

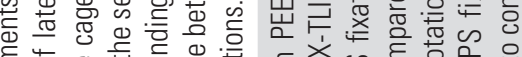

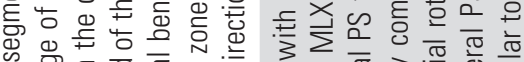

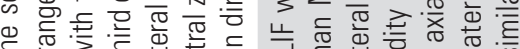

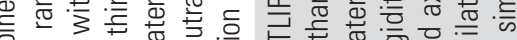

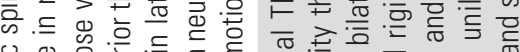
응 d心

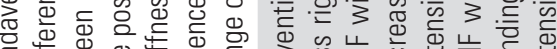

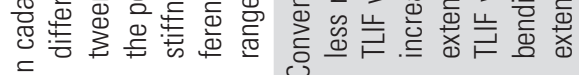

\begin{tabular}{|c|c|}
\hline & 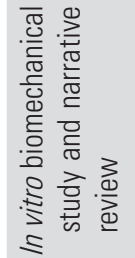 \\
\hline & 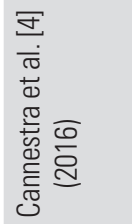 \\
\hline
\end{tabular}



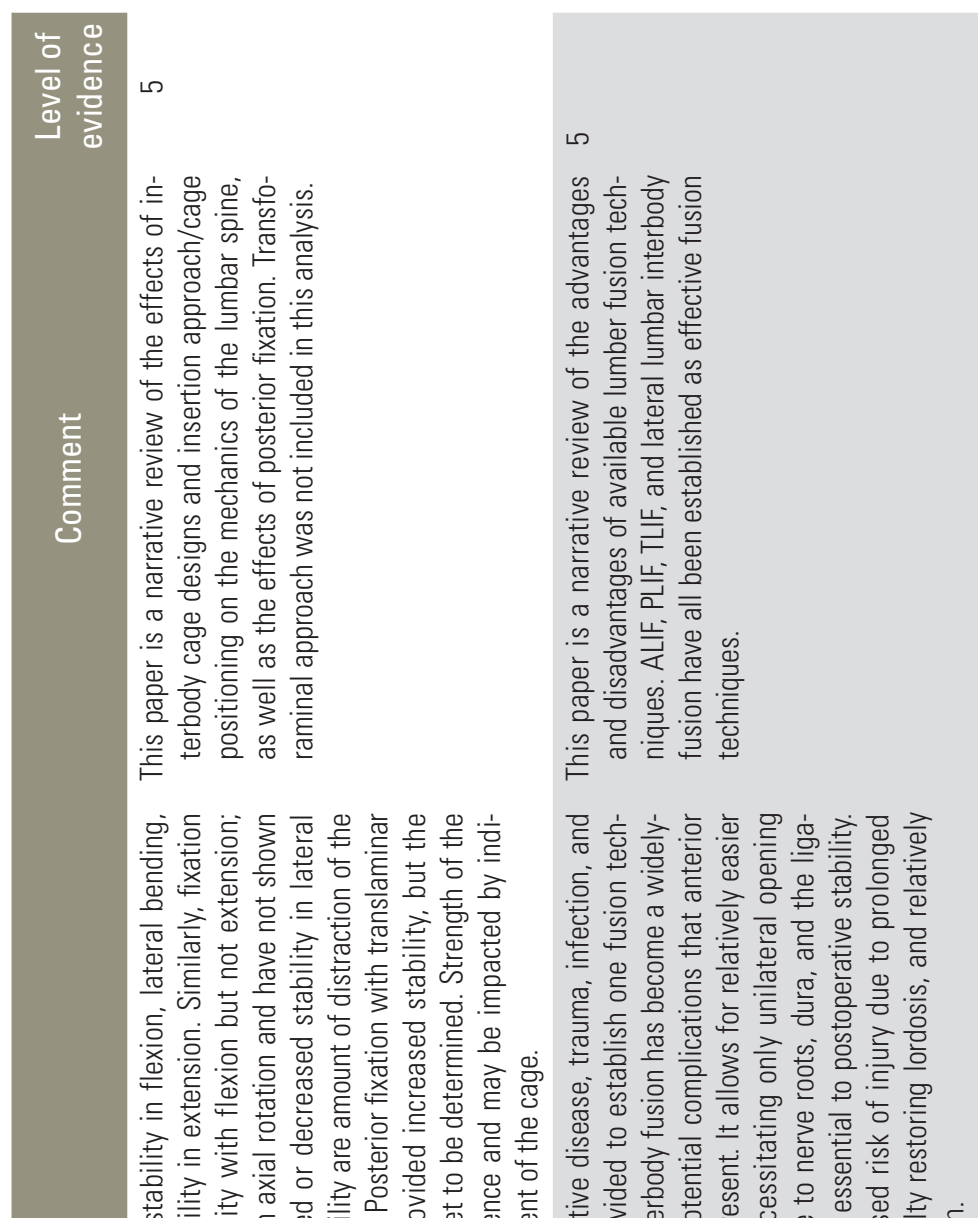

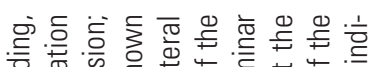

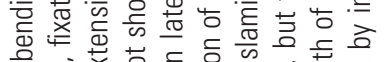

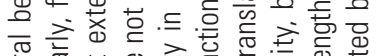

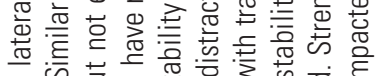

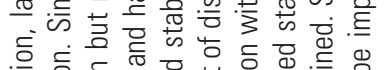
帝

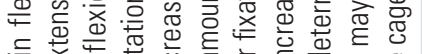

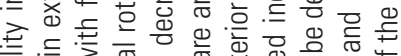

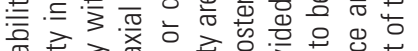

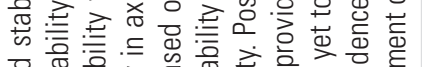

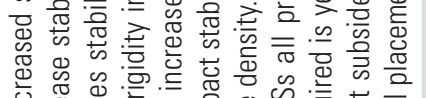

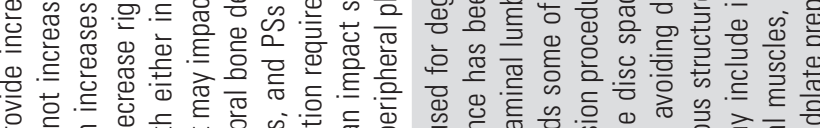

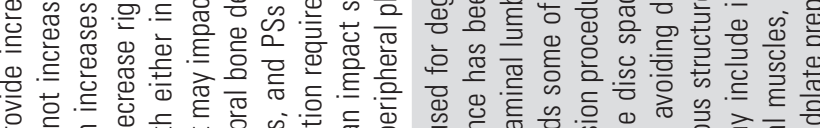

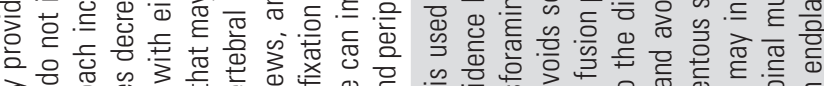

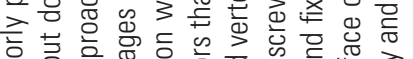

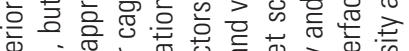

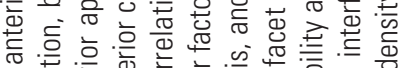

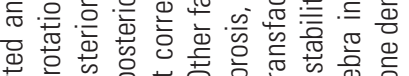

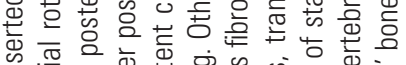

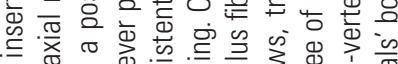

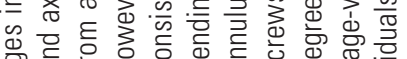

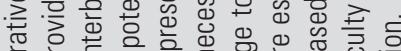

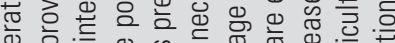

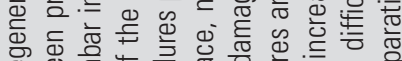

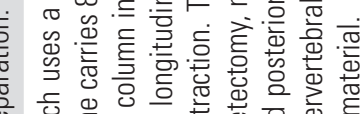

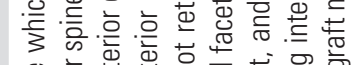

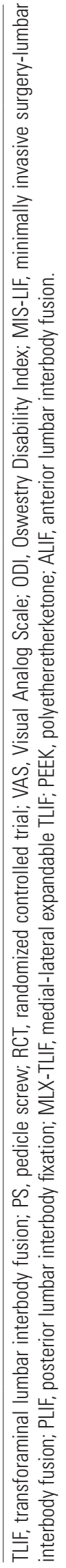
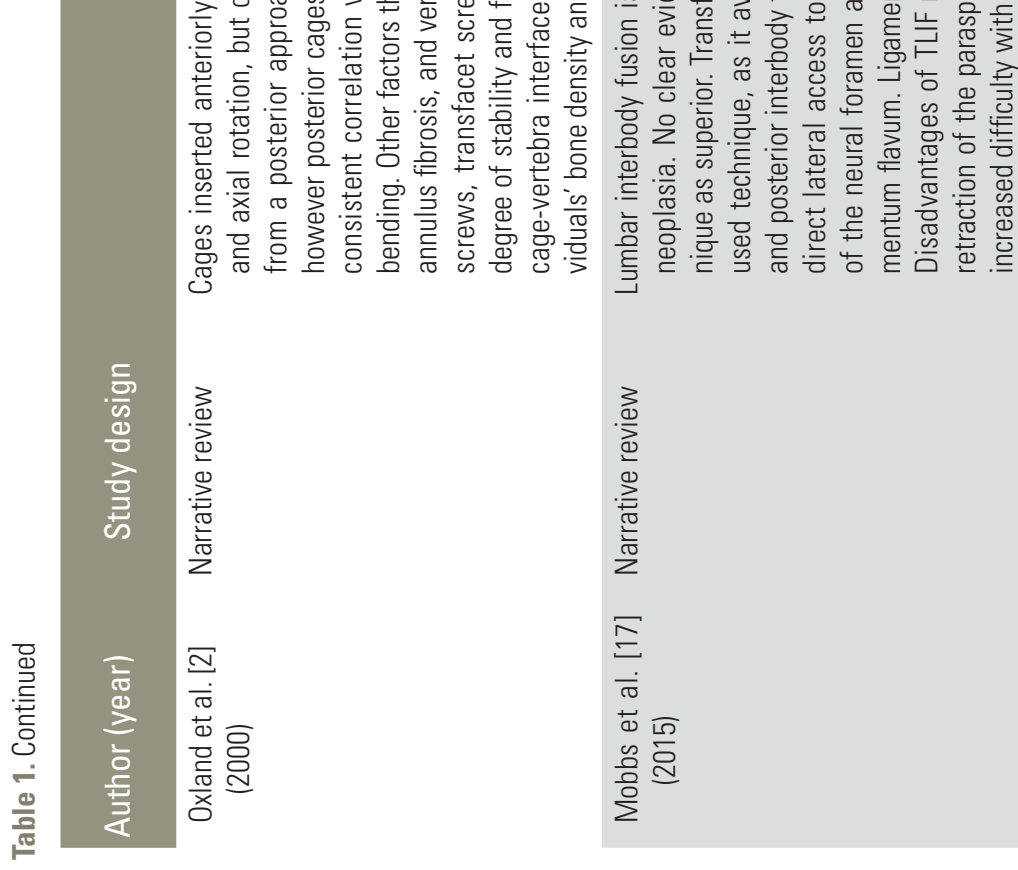

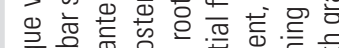

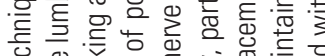

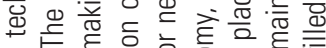

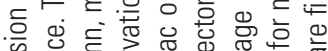

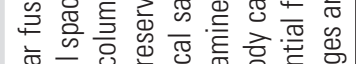

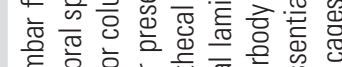

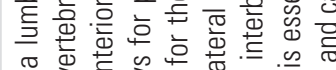

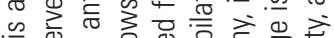

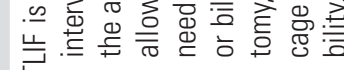
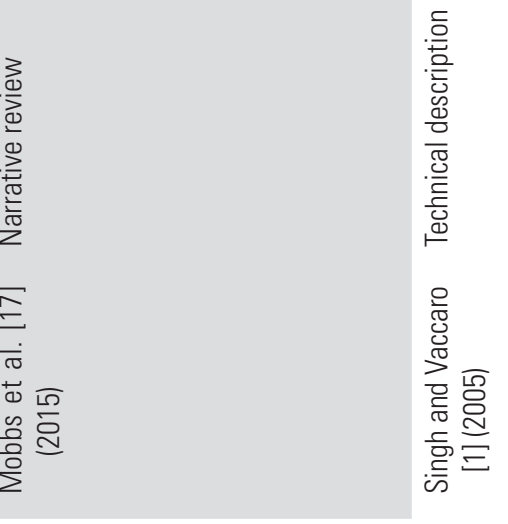
ation, and the difference in stability with the graft placed at the midline versus placed anteriorly in the sagittal plane in a single-level TLIF procedure. The authors concluded that cage position did not significantly impact the stability of the L2-L3 level and that posterior fixation with bilateral pedicle screws increased rigidity within the construct [3]. This study suggested that TLIF increased construct rigidity by the addition of posterior pedicle screw fixation, but did not address unilateral versus bilateral fixation or whether cage position impacts the method used. Similarly, an in vitro biomechanical study by Faundez et al. [16] reported no significant difference in stabilization in a comparison of semi-lunar cage placement in the anterior third of the disk space versus placement in the posterior third of the disk space and that TLIF with either approach provided significantly greater stability to the spine. Taken together, these studies suggest that the position of the cage within the sagittal plane may not impact lumbar stability with TLIF.

Oxland and Lund [2] reviewed in vitro stability following fusion with anterior and posterior approaches but excluded the transforaminal approach. Anteriorly inserted cages provided increased stability in flexion, lateral bending, and axial rotation, whereas posteriorly inserted cages increased stability with flexion. Posteriorly inserted cages also resulted in decreased rigidity in axial rotation. The authors suggested that a contributing factor to the differences could be the placement of the cage, as the cage is typically placed more centrally in the posterior approach [2]. It would therefore be worthwhile to investigate the impact of placement on stability in the transforaminal approach and also to determine whether cage position impacts stability. This review also suggested that both the anterior and posterior approaches provide inadequate stabilization of the intervertebral segment in extension [2]. If graft placement plays a role in stability, utilizing placement to maximize stability could help reduce undesirable excessive motion as well as improve clinical outcomes with unilateral screw fixation.

Castellvi et al. [13] evaluated the in vivo effects of TLIF graft position in the sagittal and coronal planes. Motion was analyzed in patients who had previously undergone TLIF surgery and then assessed in relation to the placement of their interbody graft, which was identified by radiological imaging. The anterior column of the lumbar spine carries $80 \%$ of the weight-bearing load, making it an essential component of lumbar stability and proper fu- sion [1]. The findings of this study support this assertion by showing that positioning the cage more anteriorly in the sagittal plane increased stability in flexion-extension and lateral bending. In the coronal plane, a cage placed closer to the midline provided increased stability with lateral bending [13]. These findings are indicative that TLIF cage placement may have a significant impact on lumbar stability, suggesting that stability is maximal with anterior placement. However, the study did not specify the method of posterior screw fixation, and it is unclear whether the method may have played a role in stability. Furthermore, these findings contradict those of some previous in vitro biomechanical studies $[3,16]$, underscoring the possibility that individual patient characteristics may influence postoperative stability [13]. Although this study provides a foundation for an established correlation between TLIF cage position and intervertebral stability, further exploration and data are warranted for a better understanding of this relationship.

When performing interbody fusion, a large cage footprint is desired $[4,14]$. Studies have shown that posterolateral and peripheral regions of the vertebral endplate are strongest [14] and that cages with smaller footprints placed in the weaker center of the vertebral endplate may lead to increased susceptibility to subsidence and biomechanical instability [4]. However, TLIF requires posterior access, necessitating the use of a smaller cage relative to traditional anterior lumbar interbody fusion. Tan conducted a study comparing in vitro effects of cage shape and surface area on load failure and lumbar stiffness, concluding that cage surface area did not impact rigidity. However, smaller cages did result in bone deformation beneath the cage and decreased failure load [14]. Cannestra et al. [4] found that expandable interbody cages with unilateral screw fixation provided greater stability than TLIF with a conventional banana-shaped polyetheretherketone cage with bilateral screw fixation. Although these studies offer conflicting evidence whether cage surface area directly impacts lumbar rigidity, they underscore the impact of increased surface area on the vertebral endplate to reduce subsidence, which may result in increased stability. Therefore, optimizing cage placement on the vertebral endplate in the peripheral or posterolateral position to reduce subsidence and endplate damage may result in optimal biomechanical stability. However, no definite conclusions can be drawn at this time, warranting further investigation. 
The most common site of isthmic spondylolisthesis is the L5-S1 segment, whereas the most common site of degenerative spondylolisthesis is the L4-L5 segment. Cannestra et al. [4] noted that L5-S1 and L4-L5 undergo the greatest lumbar flexion-extension, greatest shear loads, and provide two-thirds of lumbar lordosis. Cannestra et al. [4] and Harris et al. [11] reported that stability with axial rotation at L4-L5 decreased after TLIF with unilateral pedicle screw fixation compared with the intact segment, whereas Ames et al. [3] found a significant increase in stability with axial rotation at L2-L3 compared with the intact segment. These conflicting reports suggest that appropriate cage position may differ on the basis of the intervertebral level undergoing fusion due to the unique biomechanics of each level. When determining cage position, surgeons may want to consider the level at which they are operating in order to achieve optimum stability due to biomechanical effects.

Several studies included in this review found that patients undergoing fusion with unilateral pedicle screw fixation experienced less blood loss and shorter operative time relative to those undergoing bilateral fixation $[5-7,9,10]$. Many also found no significant differences in postoperative functioning, fusion rate, or complication rate between the two methods $[5-8,10]$. Based on these outcomes, unilateral pedicle screw fixation appears to be the optimal choice for patients undergoing TLIF. However, conflicting results have been reported.

In a meta-analysis of randomized control trials examining differences in clinical outcomes between patients who underwent TLIF with bilateral versus unilateral pedicle screw fixation, Xiao et al. [8] concluded that unilateral pedicle screw fixation achieved similar clinical outcomes, fusion rates, and complications as bilateral screw fixation. However, it could not be definitively concluded that unilateral fixation achieved the same efficacy and safety levels as bilateral fixation. In a systematic review and metaanalysis by $\mathrm{Hu}$ et al. [5], unilateral pedicle screw fixation yielded decreased stability compared with bilateral fixation in some cases, although ultimately the study concluded that unilateral fixation was as efficacious and safe as bilateral fixation.

In a randomized control trial by Duncan and Bailey [12] comparing rates of cage migration between patients undergoing fusion with unilateral versus bilateral fixation, there were significantly more cases of cage migration among patients undergoing unilateral screw fixation.
Similarly, Harris et al. [11] compared the stabilites of the L4-L5 lumbar segment and entire lumbar spine (T12S1) after insertion of a single carbon fiber Brantigan cage with or without posterior pedicle screw fixation. TLIF with bilateral pedicle screw fixation resulted in greater stability than TLIF with unilateral fixation, although both techniques increased stability compared with standalone cages. The authors suggested that bilateral pedicle screw fixation provided the greatest stability and should be the standard for posterior fixation following unilateral TLIF [11]. However, these studies did not address the position of the interbody cage. If the cage can be positioned to optimally stabilize the lumbar segment, this may improve the stability of the construct such that unilateral fixation is equivalent to or more stable than bilateral fixation.

Conflicting evidence in the literature has presented challenges for determining whether unilateral pedicle screw fixation provides adequate stability to be advantageous over bilateral fixation. If cage position does have a significant effect on the stability of the fusion construct, then the cage may be inserted in a position that equalizes the stability of TLIF with unilateral screw fixation to that of TLIF with bilateral screw fixation, negating the need for a second screw and minimizing invasiveness, costs, and operative time. However, as there is insufficient evidence supporting whether cage position in the intervertebral space can impact stability such that bilateral fixation is not needed, further investigation is needed.

\section{Conclusions}

To the best of our knowledge, there have been no studies evaluating the effect of cage position relative to the unilateral pedicle screw construct on the stability of the lumbar spine in TILF with unilateral pedicle screw fixation. Because greater lumbar stability after interbody fusion results in improved fusion rates, thereby leading to better clinical outcomes, we propose that additional studies testing the placement of the interbody cage position relative to the unilateral screw construct are needed to determine if ideal cage placement can render unilateral TLIF functionally equivalent to bilateral TLIF.

\section{Conflict of Interest}

No potential conflict of interest relevant to this article was reported. 


\section{Author Contributions}

Ms. Haley McKissack is the primary writer and organizer of this data. Dr. Howard Levene is the idea originator, editor, advisor, and supervisor for this work.

\section{References}

1. Singh K, Vaccaro AR. Treatment of lumbar instability: transforaminal lumbar interbody fusion. Semin Spine Surg 2005;17:259-66.

2. Oxland TR, Lund T. Biomechanics of stand-alone cages and cages in combination with posterior fixation: a literature review. Eur Spine J 2000;9 Suppl 1:S95-101.

3. Ames CP, Acosta FL Jr, Chi J, et al. Biomechanical comparison of posterior lumbar interbody fusion and transforaminal lumbar interbody fusion performed at 1 and 2 levels. Spine (Phila Pa 1976) 2005;30:E5626.

4. Cannestra AF, Peterson MD, Parker SR, Roush TF, Bundy JV, Turner AW. MIS expandable interbody spacers: a literature review and biomechanical comparison of an expandable MIS TLIF with conventional TLIF and ALIF. Spine (Phila Pa 1976) 2016;41 Suppl 8:S44-9.

5. Hu XQ, Wu XL, Xu C, et al. A systematic review and meta-analysis of unilateral versus bilateral pedicle screw fixation in transforaminal lumbar interbody fusion. PLoS One 2014;9:e87501.

6. Liu Z, Fei Q, Wang B, et al. A meta-analysis of unilateral versus bilateral pedicle screw fixation in minimally invasive lumbar interbody fusion. PLoS One 2014;9:e111979.

7. Chen C, Cao X, Zou L, Hao G, Zhou Z, Zhang G. Minimally invasive unilateral versus bilateral technique in performing single-segment pedicle screw fixation and lumbar interbody fusion. J Orthop Surg Res 2015;10:112.

8. Xiao SW, Jiang H, Yang LJ, Xiao ZM. Comparison of unilateral versus bilateral pedicle screw fixation with cage fusion in degenerative lumbar diseases: a metaanalysis. Eur Spine J 2015;24:764-74.
9. Lin B, Xu Y, He Y, Zhang B, Lin Q, He M. Minimally invasive unilateral pedicle screw fixation and lumbar interbody fusion for the treatment of lumbar degenerative disease. Orthopedics 2013;36:e1071-6.

10. Zhang K, Sun W, Zhao CQ, et al. Unilateral versus bilateral instrumented transforaminal lumbar interbody fusion in two-level degenerative lumbar disorders: a prospective randomised study. Int Orthop 2014;38:111-6.

11. Harris BM, Hilibrand AS, Savas PE, et al. Transforaminal lumbar interbody fusion: the effect of various instrumentation techniques on the flexibility of the lumbar spine. Spine (Phila Pa 1976) 2004;29:E6570.

12. Duncan JW, Bailey RA. An analysis of fusion cage migration in unilateral and bilateral fixation with transforaminal lumbar interbody fusion. Eur Spine J 2013;22:439-45.

13. Castellvi AD, Thampi SK, Cook DJ, et al. Effect of TLIF cage placement on in vivo kinematics. Int J Spine Surg 2015;9:38.

14. Tan JS, Bailey CS, Dvorak MF, Fisher CG, Oxland TR. Interbody device shape and size are important to strengthen the vertebra-implant interface. Spine (Phila Pa 1976) 2005;30:638-44.

15. Kim JY, Park JY, Kim KH, et al. Minimally Invasive transforaminal lumbar interbody fusion for spondylolisthesis: comparison between isthmic and degenerative spondylolisthesis. World Neurosurg 2015;84:1284-93.

16. Faundez AA, Mehbod AA, Wu C, Wu W, Ploumis A, Transfeldt EE. Position of interbody spacer in transforaminal lumbar interbody fusion: effect on 3-dimensional stability and sagittal lumbar contour. J Spinal Disord Tech 2008;21:175-80.

17. Mobbs RJ, Phan K, Malham G, Seex K, Rao PJ. Lumbar interbody fusion: techniques, indications and comparison of interbody fusion options including PLIF, TLIF, MI-TLIF, OLIF/ATP, LLIF and ALIF. J Spine Surg 2015;1:2-18. 